\title{
Exciton Dephasing in Quantum Dot Molecules
}

\author{
P. Borri, W. Langbein, U. Woggon, M. Schwab, and M. Bayer \\ Experimentelle Physik II, Universität Dortmund, Otto-Hahn Strasse 4, D-44227 Dortmund, Germany
}

\author{
S. Fafard, Z. Wasilewski, and P. Hawrylak \\ Institute for Microstructural Science, National Research Council, Ottawa, K1A OR6, Canada
}

(Received 29 July 2003; published 23 December 2003)

\begin{abstract}
We have measured the exciton dephasing time in InAs/GaAs quantum dot molecules having different interdot barrier thicknesses in the temperature range from 5 to $60 \mathrm{~K}$, using a highly sensitive four-wave mixing heterodyne technique. At $5 \mathrm{~K}$ dephasing times of several hundred picoseconds are found. Moreover, a systematic dependence of the dephasing dynamics on the barrier thickness is observed. These results show how the quantum-mechanical coupling of the electronic wave functions in the molecules affects both the exciton radiative lifetime and the exciton-acoustic phonon interaction.
\end{abstract}

DOI: 10.1103/PhysRevLett.91.267401

The coherent optical manipulation of the fundamental interband transition involving Coulomb-correlated valence and conduction band states (ground-state excitonic transition) in semiconductor quantum dots (QDs) is currently receiving a lot of attention, beyond its fundamental interest, for its possible applications in the field of quantum computing [1]. Usually, one describes the interaction with the environment of a quantum-mechanical two-level system, which serves as a quantum bit (qubit), in the form of dephasing processes. Decoherence due to dephasing is a fundamental obstacle to overcome in quantum computation.

It was recently found that the dephasing time of the ground-state excitonic transition at low temperatures $(\sim 5 \mathrm{~K})$ in epitaxially grown InGaAs/GaAs selfassembled quantum dots is in the nanosecond range $[2,3]$. Moreover, it has been demonstrated experimentally [4-6] that a basic single-qubit operation such as a resonant $\pi$ Rabi rotation can be performed on a time scale ( $\sim 1 \mathrm{ps})$ much shorter than the dephasing time, due to the large transition dipole moment in these systems, making InGaAs/GaAs QDs appealing for quantum computing.

Consequently, there is a significant interest in using "artificial molecules" formed by two quantum mechanically coupled QDs for the realization of quantum gates involving two-qubit operations [7-9]. In such systems, the coupling involves tunneling of electrons and holes between two adjacent dots separated by a thin barrier region of thickness $d$. The ground-state excitonic transition splits into two optically active transitions which increase their energy separation with decreasing $d$. The energy splitting is determined by the interplay between single particle tunneling and Coulomb interactions. Quantum dot molecules (QDMs) can be fabricated by vertically stacking two layers of epitaxially grown selfassembled QDs. It is, however, technologically challenging to obtain resonant quantum-mechanical coupling due to size, composition, and strain inhomogeneities inherent in the growth of QD ensembles. Recent advance-
PACS numbers: 78.67.Hc, 42.50.Md, 63.22.+m, 78.47.+p

ments in the growth technology have enabled the fabrication of InAs/GaAs QDMs exhibiting quantummechanical coupling with an energy splitting of several tens of meV, comparable to the inhomogeneous broadening [10]. While a lot of work was devoted so far to study the excitonic transition energies by photoluminescence spectroscopy in these structures [9-12], no investigations have yet been reported on the exciton dephasing in QDMs, despite its fundamental importance.

In this Letter, we report the exciton dephasing time measured at low temperatures in a series of QDMs with variable interdot barrier thickness $d$, using a highly sensitive four-wave mixing (FWM) heterodyne technique. At $5 \mathrm{~K}$ we measure long dephasing times, in the range of several hundred picoseconds. Moreover, we find that the quantum-mechanical coupling of the electronic wave functions between adjacent dots influences the exciton dephasing and its temperature dependence. Systematic variations versus $d$ are found which are of key importance in tailoring QDMs for applications involving the coherent manipulation of excitonic transitions in these systems.

The investigated samples are of the same type as in Refs. $[9,11]$ where quantum-mechanical coupling of individual molecules was shown. They consist of two layers of vertically stacked InAs/GaAs QDs with an areal dot density of $\sim 10^{10} \mathrm{~cm}^{-2}$. The QD shape is approximately cylindrically symmetric with radius of $8-12 \mathrm{~nm}$ and height of 1-2 $\mathrm{nm}$. We studied five different samples with nominal distances $d$ (from wetting layer to wetting layer) ranging from 16 to $5 \mathrm{~nm}$ and a reference sample containing only a single QD layer. In Fig. 1 photoluminescence spectra are shown for an ensemble of QDMs with $d=5 \mathrm{~nm}$ and for the single QD layer, measured at $2 \mathrm{~K}$ using nonresonant laser excitation of different intensities $\left(I_{0}\right)$. The spectrum at $I_{0}=40 \mathrm{~W} / \mathrm{cm}^{2}$ for the single QD layer shows the energy distribution in the QD ensemble of the ground-state transition involving the excitonic recombination from the two lowest-lying electron 


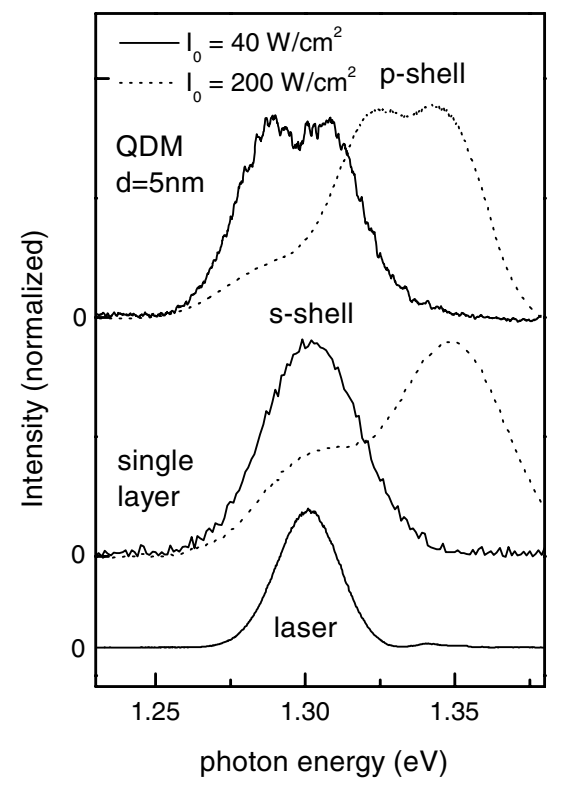

FIG. 1. Photoluminescence spectra at $2 \mathrm{~K}$ of an ensemble of InAs/GaAs QDMs with $5 \mathrm{~nm}$ nominal barrier thickness and of a single layer of QDs, at different excitation intensities $I_{0}$ as indicated. The spectrum of the laser pulse of $100 \mathrm{fs}$ duration used in the four-wave mixing experiment is also shown.

and hole states (so-called $s$ shell), from which we infer a Gaussian inhomogeneous broadening of $37 \mathrm{meV}$ full width at half maximum (FWHM). With increasing excitation, a second group of excitonic transitions appears, $\sim 47 \mathrm{meV}$ above the ground-state transition, involving the recombination of electrons and holes in their first respective excited state (so-called $p$ shell). On the QDMs with $d=5 \mathrm{~nm}$ a transition doublet for both the $s$-shell and $p$-shell recombination is visible, indicating that the majority of dots is quantum mechanically coupled, with an inhomogeneous broadening of transition energies and coupling strengths in the ensemble [11]. From the energy splitting of the $s$-shell doublet, we infer $\sim 23 \mathrm{meV}$ energy separation between the two optically active excitonic transitions [13].

The FWM experiment is performed using a Ti:sapphire laser providing Fourier-limited pulses of 100 fs duration at $76 \mathrm{MHz}$ repetition rate, in resonance with the center of the inhomogeneously broadened excitonic ground-state transitions, as shown in Fig. 1. Two exciting pulses 1,2 with variable relative delay time $\tau$ propagate along two different incident directions $\vec{k}_{1,2}$, with pulse 1 leading pulse 2 for $\tau>0$. Quarter-wave plates were used for polarization control of the pulses. The FWM signal was detected along $2 \vec{k}_{2}-\vec{k}_{1}$ in transmission geometry. The excitation intensities where chosen to give rise to FWM in the third-order regime. In order to distinguish FWM intensities down to 15 orders of magnitude lower in intensity than the transmitted beams along $\vec{k}_{1,2}$, we additionally used a heterodyne detection technique similar to the one discussed in our previous work [2]. In this technique, the pulse 1 (2) is frequency shifted by a radio frequency $\omega_{1}\left(\omega_{2}\right)$, and the interference of the FWM signal with an unshifted reference pulse is detected at $2 \omega_{2}-\omega_{1}$ using high-frequency photodiodes and a lock-in amplifier. In this way, the FWM field amplitude is measured via its interference with the field of the reference pulse. The samples were antireflection coated to reduce multiple reflections and increase light coupling and were held in a cryostat at temperatures varying from 5 to $60 \mathrm{~K}$.

The time-integrated FWM field amplitude versus $\tau$ is shown in Fig. 2 for the single QD layer and for the QDMs with $d=6 \mathrm{~nm}$. On the single QD layer at $5 \mathrm{~K}$ the dynamics is dominated by a long decay time, from which we infer a dephasing time of $T_{2}=590 \pm 10 \mathrm{ps}$. In the initial dynamics, a fast decay within few ps is also visible. As discussed in Ref. [2] this nonexponential FWM decay corresponds to a non-Lorentzian homogeneous line shape with a sharp zero-phonon line (ZPL) and a broad band from elastic exciton-acoustic phonon interaction. A damped oscillation is also present in the dynamics at long delays. When the cylindrical symmetry is lowered, InGaAs QDs exhibit a fine-structure splitting of the ground-state excitonic transition due to the anisotropic exchange interaction, resulting into two linearly polarized bright transitions separated in energy by a few tens of $\mu \mathrm{eV}$ [14]. The observed oscillation arises from the beating between the optically driven polarizations of the two bright excitonic transitions having oscillation frequencies separated by the fine-structure splitting (note that $30 \mu \mathrm{eV}$ splitting corresponds to a beat period of $140 \mathrm{ps}$ ). The inhomogeneous distribution of fine-structure splitting energies in the ensemble results in a destructive interference and thus in a damping in the oscillation

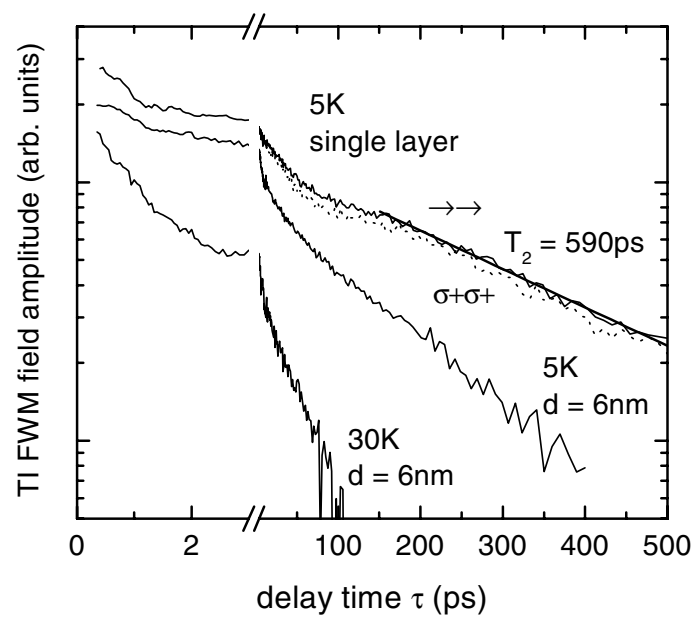

FIG. 2. Time-integrated four-wave mixing field amplitude on a single layer of quantum dots and on quantum dot molecules with $d=6 \mathrm{~nm}$, at different temperatures as indicated. The solid (dotted) curves were obtained using colinearly (cocircularly) polarized exciting pulses. An exponential fit to the FWM decay dynamics at long delays on the single QD layer is also shown and the corresponding dephasing time is indicated. 
amplitude versus delay [15]. This interpretation is supported by a more pronounced beating amplitude for cocircularly polarized beams (dotted trace in Fig. 2) exciting a balanced mixture of the two linearly polarized transitions.

On the QDMs, we distinguish three major differences in the FWM dynamics at $5 \mathrm{~K}$ as compared to the single QD layer. (i) The dynamics at long delay times is faster $\left(T_{2}=340 \mathrm{ps}\right)$, (ii) it is strongly nonexponential, and (iii) the fast initial dynamics has smaller amplitude. With increasing temperature the dephasing time decreases and the relative amplitude of the initial dynamics increases, as shown in Fig. 2 at $30 \mathrm{~K}$. We should comment that on the QDMs one would expect to observe a beating in the FWM dynamics from the interference of the polarizations of the two excitonic transitions which form the $s$-shell doublet. An energy separation of $23 \mathrm{meV}$ between these two transitions, as observed for the QDMs with $d=$ $5 \mathrm{~nm}$ (see Fig. 1), corresponds to a beat period of only $180 \mathrm{fs}$. This cannot be resolved in our experiment since the first $\sim 300 \mathrm{fs}$ dynamics is dominated by nonresonant FWM in the GaAs barrier. For QDMs with larger $d$ an increase of the beat period up to $\sim 500 \mathrm{fs}$ is expected [9]. However, the inhomogeneous distribution of energy separations is causing a rapid decay of the beating and hindered its observation.

The above mentioned differences between QDMs and QDs are quantified in Fig. 3 where the temperature dependence of the homogeneous broadening $\gamma$ (FWHM) corresponding to the measured dephasing time, and of the weight of the ZPL, is shown for the single QD layer and for the QDMs with $d=6 \mathrm{~nm}$. In the QDMs the FWM decay at long delays was fitted with two exponential decay times, and thus two values $\gamma_{a}, \gamma_{b}$ are shown in the figure. The ZPL weight (i.e., the ratio in the absorption line shape between the area of the ZPL and the total area of the line) was inferred by the ratio between the amplitude of the FWM field at $\tau \sim 0$ and the amplitude of the FWM field at long delays extrapolated for $\tau \rightarrow 0$, according to the expression in Ref. [16]. The temperature dependence of $\gamma_{b}$ was fitted with a constant term $\left(\gamma_{0}\right)$ and an activated part, according to the formula indicated in the figure. The homogeneous broadening as well as the ZPL weight is higher in QDMs than in QDs at all investigated temperatures.

The changes of the measured excitonic dephasing and its temperature dependence going from uncoupled QDs to QDMs is summarized in Fig. 4 where we plotted the zerotemperature homogeneous broadening $\gamma_{0}$, the activation energy $E_{\mathrm{A}}, \gamma_{a}($ at $10 \mathrm{~K})$, and the ZPL weight (at $10 \mathrm{~K}$ ) versus barrier thickness $d$. Remarkably, all four parameters exhibit a systematic dependence versus $d$ [17]. In particular, $\gamma_{0}, \gamma_{a}$ and the ZPL weight increase with decreasing $d$ while $E_{\mathrm{A}}$ decreases.

A quantitative understanding of the measured dependencies requires a calculation of the electronic energy levels, of the exciton-acoustic phonon interaction, and

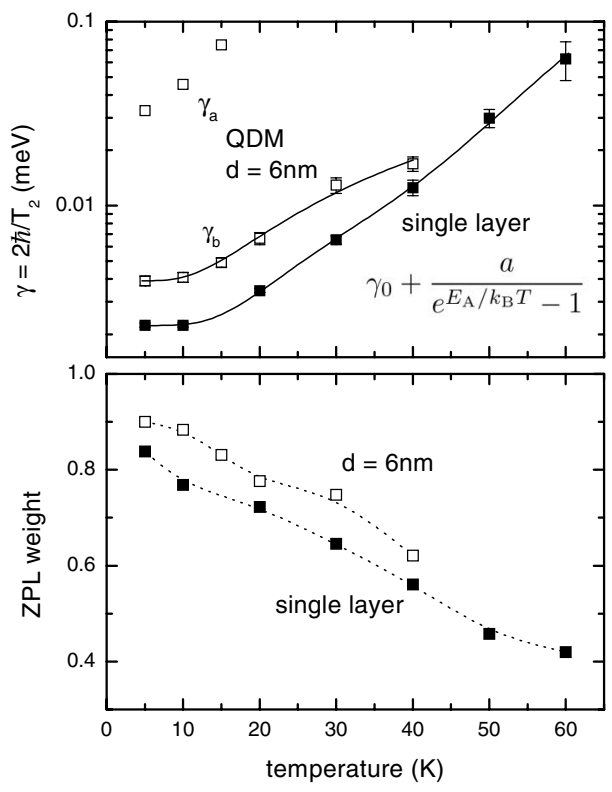

FIG. 3. Top: Homogeneous broadening $\gamma$ versus temperature on a single layer of quantum dots and on quantum dot molecules with $d=6 \mathrm{~nm}$. For the QDMs, $\gamma_{a}, \gamma_{b}$ correspond to the two exponential decay times in the FWM decay. Solid lines are fits to the data according to the indicated formula. Bottom: Weight of the zero-phonon line versus temperature. Dotted lines are guides for the eye.

of the exciton oscillator strength versus $d$ in QDMs, which is beyond the scope of this Letter. We propose the following qualitative interpretation.

The increase of $\gamma_{0}$ with decreasing $d$ is consistent with an increased exciton oscillator strength, and thus radiative broadening, in QDMs that have larger exciton coherence volume than uncoupled QDs [18]. However, the measured increase is more than a factor of 2, while the superradiant excitonic transition in the QDM would have at most twice the radiative broadening of the uncoupled QD case.

The increase of $E_{\mathrm{A}}$ with increasing $d$ could be explained when interpreting $E_{\mathrm{A}}$ as the energy separation between the ground-state excitonic transition and the excitonic excited state involving the first excited hole level, as we suggested in Ref. [2]. In fact, since an excited-state wave function penetrates more in the barrier than the wave function of the ground state, the hole excited state undergoes a larger tunneling splitting than the ground state with decreasing $d$ and thus its energy separation to the ground-state level of the same symmetry decreases. However, it has been recently proposed that an activated temperature dependence of the ZPL homogeneous broadening can also be explained by phononphonon scattering with an activation energy of the lowest Brillouin-zone-edge phonon frequency [19]. This interpretation is indeed supported by measurements (not shown here) on a series of QDs with different confinement energies which showed an activation energy independent of the separation between the electronic energy levels. 


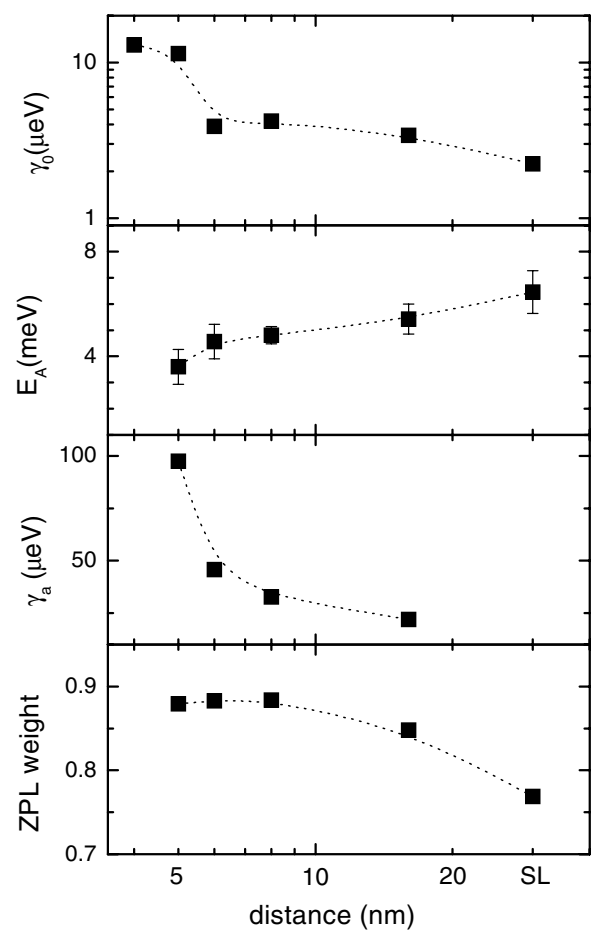

FIG. 4. Dependence on the barrier thickness $d$ of the following: zero temperature extrapolated homogeneous broadening $\gamma_{0}$, the activation energy $E_{\mathrm{A}}$, the homogeneous broadening $\gamma_{a}$ resulting from the fast decay in the nonexponential FWM dynamics in QDMs at $10 \mathrm{~K}$, and the weight of the zero-phonon line at $10 \mathrm{~K}$. The data of the single layer (SL) are also shown for comparison.

The strong temperature dependence of $\gamma_{a}$ (see Fig. 3) and its dependence on $d$ support an attribution of this broadening as due to the phonon relaxation mechanisms from the bright exciton state of higher energy in QDMs, rather than an effect of the inhomogeneously broadened fine-structure splitting [11]. Since the energy separation between the two bright exciton states increases with decreasing $d$, the density of phonon states available for this relaxation increases and thus also $\gamma_{a}$. This interpretation is supported by the observation of a higher amplitude of the decay component associated to $\gamma_{a}$ in the FWM dynamics when we excited more resonantly to the higher peak of the $s$-shell doublet (not shown here).

Finally, the increase of the ZPL weight with decreasing $d$ is consistent with an increase of the extension of the exciton wave function, and thus of the coherence volume, in QDMs. In fact, the presence of a broad acoustic phonon band superimposed to the ZPL in the homogeneous line shape is directly connected with the local character of the electronic excitation in QDs giving rise to a local lattice distortion [20]. The less the exciton wave function is localized, the less important the acoustic phonon band in the line shape, i.e., the larger the ZPL weight.

In conclusion, our experimental data indicate that both the exciton radiative broadening and the exciton-acoustic phonon interaction systematically change, going from uncoupled quantum dots to quantum dot molecules. These findings should stimulate advancements in the theoretical understanding of these fundamental processes in semiconductor nanostructures. For the possible applications of quantum dot molecules for quantum operations, our results show that while quantum dot molecules exhibit faster dephasing with increasing quantum-mechanical coupling, still the dephasing time at low temperature is in the $\sim 100 \mathrm{ps}$ time scale, much longer than the period of a coherent $\pi$ Rabi rotation, especially when taking into account an increased exciton oscillator strength in these systems. Moreover, the increased weight of the zero-phonon line indicates that the phonon-assisted damping is reduced in quantum dot molecules and that they tend to recover a Lorentzian absorption line shape as compared to uncoupled dots.

This work was supported by DFG (Wo477/17-1, Ba1549/7-1, and GK726) and by the NRC-Helmholtz Science and Technology fund. P. B. is supported by the European Union (HPMF-CT-2000-00843).

[1] Pochung Chen, C. Piermaroccchi, and L. J. Sham, Phys. Rev. Lett. 87, 067401 (2001).

[2] P. Borri et al., Phys. Rev. Lett. 87, 157401 (2001).

[3] D. Birkedal, K. Leosson, and J. M. Hvam, Phys. Rev. Lett. 87, 227401 (2001).

[4] P. Borri et al., Phys. Rev. B 66, 081306(R) (2002).

[5] A. Zrenner et al., Nature (London) 418, 612 (2002).

[6] H. Htoon et al., Phys. Rev. Lett. 88, 087401 (2002).

[7] Guido Burkard, Daniel Loss, and David P. DiVincenzo, Phys. Rev. B 59, 2070 (1999).

[8] Gang Chen et al., Science 289, 1906 (2000).

[9] M. Bayer et al., Science 291, 451 (2001).

[10] S. Fafard, M. Spanner, J. P. McCaffrey, and Z. R. Wasilewski, Appl. Phys. Lett. 76, 2268 (2000).

[11] G. Ortner et al., Phys. Rev. Lett. 90, 086404 (2003).

[12] I. Shtrichman et al., Phys. Rev. B 65, 081303 (2002).

[13] Photoluminescence spectra were measured in all investigated ensembles and showed a systematic increase of the splitting with decreasing $d$.

[14] M. Bayer et al., Phys. Rev. Lett. 82, 1748 (1999).

[15] W. Langbein et al., Phys. Rev. B 55, R7383 (1997).

[16] A. Vagov, V. M. Axt, and T. Kuhn, Phys. Rev. B 67, 115338 (2003).

[17] The parameter $a$ was in the range of $50 \mu \mathrm{eV}$ and was not systematically varying versus $d$.

[18] Population lifetimes on the QDMs have been measured using time-resolved photoluminescence and show a similar trend versus $d$ as $\gamma_{0}$.

[19] R. Zimmermann and E. Runge, in Proceedings of the 26th International Conference on the Physics of Semiconductors, edited by J. H. Davies and A. R. Long (Institute of Physics Publishing, Bristol, UK, 2002).

[20] A. Vagov, V. M. Axt, and T. Kuhn, Phys. Rev. B 66, 165312 (2002). 